\title{
Influence of suspension parameter for derailment analysis of a full railway vehicle model cruising on a curved track
}

\author{
Yamika Patel*, Vikas Rastogi and Wolfgang Borutzky \\ Department of mechanical Engineering, Delhi Technological University, Delhi \\ Corresponding Author : yamika_phd2k17@dtu.ac.in
}

\begin{abstract}
The main intention of this research work is to study the derailment response of high speed railway vehicle (HSRV) cruising on a curved track. In previous research work, lower degree of freedom (DOF) has been considered for the derailment analysis which may not give more accurate results. Hence, a 31 DOF bondgraph model of HSRV has been developed which consist of carbody, two truck frames and two selfsame wheelsets for each truck frame. Vertical, lateral, roll, yaw and pitch motion are considered for carbody and bogie and except pitch motion all the other motion are considered for wheelsets. Non-linearities in terms of heuristic nonlinear creep model and flange contact has been employed to simulate the derailment response at high speed. The effect of vehicle speed running on a curved track was investigated for derailment quotient. The main aim of present research work to evaluate derailment quotient at the speed range of $150 \mathrm{kmph}$ to $600 \mathrm{kmph}$ for hard and soft suspension parameter. Derailment quotient has been calculated for both linear and nonlinear creep models and it is seen that DQ for linear model has a lower value compare to non linear creep. The major advantages of the proposed model are that, the presented model can actively predict the derailment of a railway vehicle, and also precisely determine the nonlinear critical hunting speeds.
\end{abstract}

Keywords: Heuristics non linear creep model; Suspension element; Derailment; Flange contact.

\section{INTRODUCTION}

With the advent in speed, the safety of a high-speed railway vehicle, particularly its derailment behaviour, is a very important issue. Vehicle designers have always been interested in the issue of high speed with no derailment. In the past few years, a number of researches have been administered to resolve this issue. During a derailment, the wheel drifts away from the rail and is unable to continue rolling. To analyze this effect, Nadal proposed a derailment ratio, which solely depends on lateral and vertical forces acting on wheel, flange contact, friction coefficient between wheel and rail (F.J Nadal., 1896). However, Weinstock studied that derailment was associated not only with the derailment coefficient on the flange side but also at the non-contact side of the flange (H Weinstock.,1984). From experiment, it is evaluated that above mentioned criteria are valid only for small yaw angle (L. Sweet et al., 2018). Under quasistatic assumption, Barbosa developed a 3D contact model to improve Nadal derailment criteria by considering creep forces (R. Barbosa., 2004). A lot of literature study has been found on lateral stability of high speed railway vehicle which considered the linear and non linear creep forces (C.E Bell et al., 1981)(Y.C Cheng et al., 2011)(R.V Dukkipati et al., 2001). Wickens provided the curving performance of the asymmetrical truck on the basis of linear creep model neglecting the creep moments between wheels and rails (A.H Wickens., 1996). Using oblique impact theory, Polach and Wang determine the critical hunting speed for impact derailment (W Wang et al., 2012). 
From the work of Zboinski and Dusza, the creep moments were estimated from the longitudinal and lateral creep forces on the basis of longitudinal, lateral and spin creepages (K. Zboinski et al., 2006). In some of the researches, the nonlinear creep moment were neglected while using the nonlinear creep models (K. Zboinski et al., 2006)(J. Zeng et al., 2004)(O Polach., 2006)(O Polach., 1999)(Y.C Cheng et al., 2009). Also, the derailment analysis of various vehicle models was carried out by utilising the linear creep model. Under practical scenario, the hunting stability of railway vehicles and their dynamic response is sensitive to the method of calculating creep forces and moments. Cheng et al. constructed a 20 DOF model accounting the lateral displacement and yaw angle for each wheelset, lateral and vertical displacements for bogie and car body, and roll and yaw angles for bogie and car body (Y.C Cheng et al., 2009). While constructing the 20 DOF model, Cheng et al. neglected the roll angle of each wheelset. But in practical scenario, roll angle is considered as an important DOF of each wheelset for determining the contact forces between rails and wheels. Hence, the determination of derailment quotient may get wronged if the roll angle of each wheelset is neglected.

Despite the fact, Wang et al. has estimated the derailment quotient using the complicated railway vehicle models, no study has been conducted yet so far for evaluating the derailment quotient for both the linear and nonlinear creep models along with the suspension parameters (W Wang et al., 2010).

In this study, the roll angle of wheelsets is considered while constructing a 31-DOF model. This provides an accurate evaluation of derailment quotient. The model was created using BONDGRAPH simulation technique. This modelling technique has advantage that the suspension forces are intrinsically available in BONDGRAPH modelling technique as suspension elements. A 31-DOF model is created for the analysing the derailment behaviour of a highspeed railway vehicle. This 31 DOF model considers the pitch motion, lateral, vertical, yaw and roll motion for, vehicle body and two bogies but for four selfsame wheelsets, except pitch motion all other motions were considered for investigating the derailment quotient. The Derailment quotient was investigated under the condition when the railway vehicle is running on the curved track with speed varying from $150 \mathrm{Kmph}$ to $600 \mathrm{Kmph}$. This 31 -DOF model uses the heuristic nonlinear creep model to evaluate the derailment quotient while considering both nonlinear creep forces and moments. This study helps to get the complete behaviour of a high speed railway vehicle running on a curved track from the view point of derailment incidences occurred in real world. The benefits of using the presented model are that it can provide accurate values of derailment quotient, safe speed of vehicle running on the curved tracks. This evaluated data will foretell the occasion of derailment before it happened.

\section{DESCRIPTION OF BONDGRAPH MODEL OF 31-DOF HIGH SPEED RAILWAY VEHICLE SYSTEM AND ITS GOVERNING EQUATIONS}

Each model of railway vehicle is integration of three parts: a car body, bogies and two selfsame wheel sets. These various parts are joined together via a suspension system placed in longitudinal, lateral and vertical direction. A primary suspension system is mounted in between the selfsame wheel set and truck frame. Similarly, the secondary suspension system is mounted in between truck frame and car body as shown in Figure 1. The components of railway model are taken as rigid mass and are portrayed by mass of inertia. By considering all the parts of railway vehicle and the suspension system between them, a 31 DOF railway model is created for the derailment analysis of the railway system. For the creation of a model, a building block way of model creation is used. In Building block way, every part of the railway vehicle system is modelled separately and then all the parts were put together in an order to create the integrated model of railway vehicle system. 


\section{STRUCTURE OF SUB-MODEL SYSTEM}

A railway vehicle is integration of various subparts; a vehicle car body structure, truck frame and 2-selfsame wheelsets for each truck frame. These parts of a railway vehicle are interconnected through a suspension system in vertical, lateral and longitudinal direction. A primary suspension system is incorporated between a bogey and 2-selfsame wheelsets whereas a secondary suspension system is incorporated between bogey and vehicle car body. The complete suspension system is modelled through the combination of springs and damping elements having motion in all 3-coordinate axes. With all the possible motions, the proposed system is having a total of $31 \mathrm{DOF}$. The proposed model is developed by using Building Block approach. In this approach, all the parts are modelled separately and then integrated together to complete the model. Figure 1 illustrates the railway vehicle model.

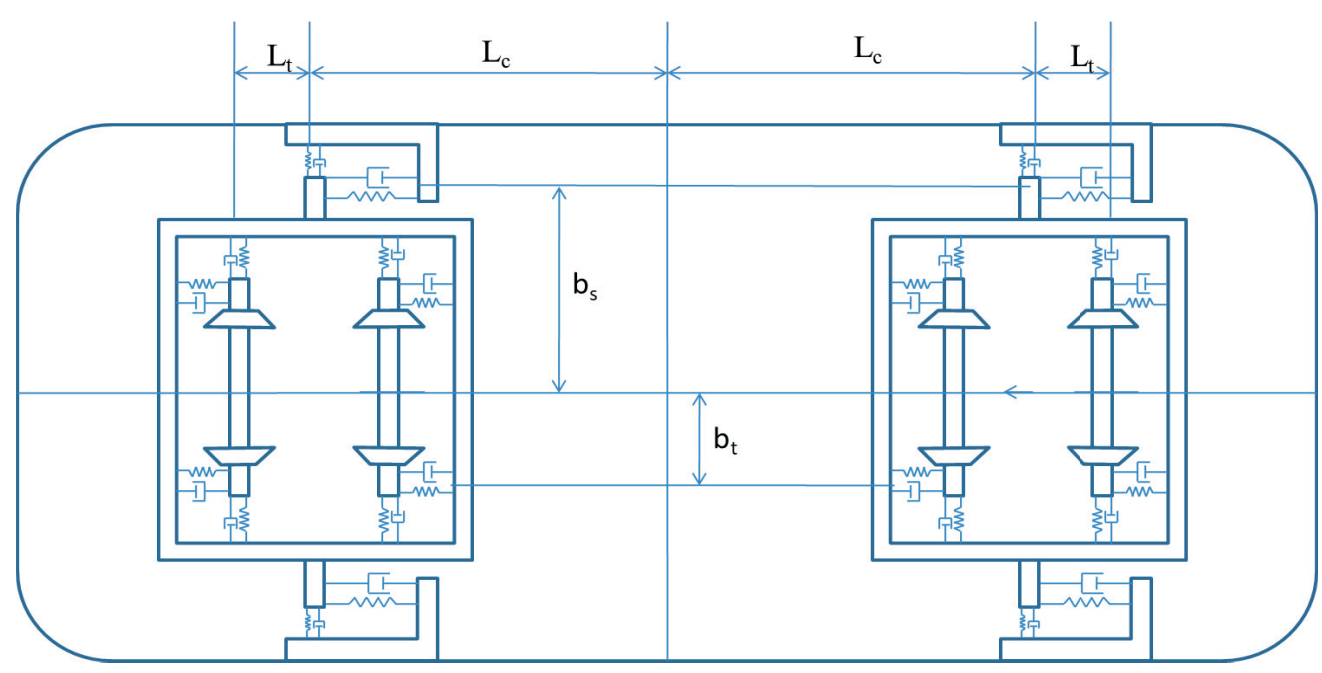

(a)

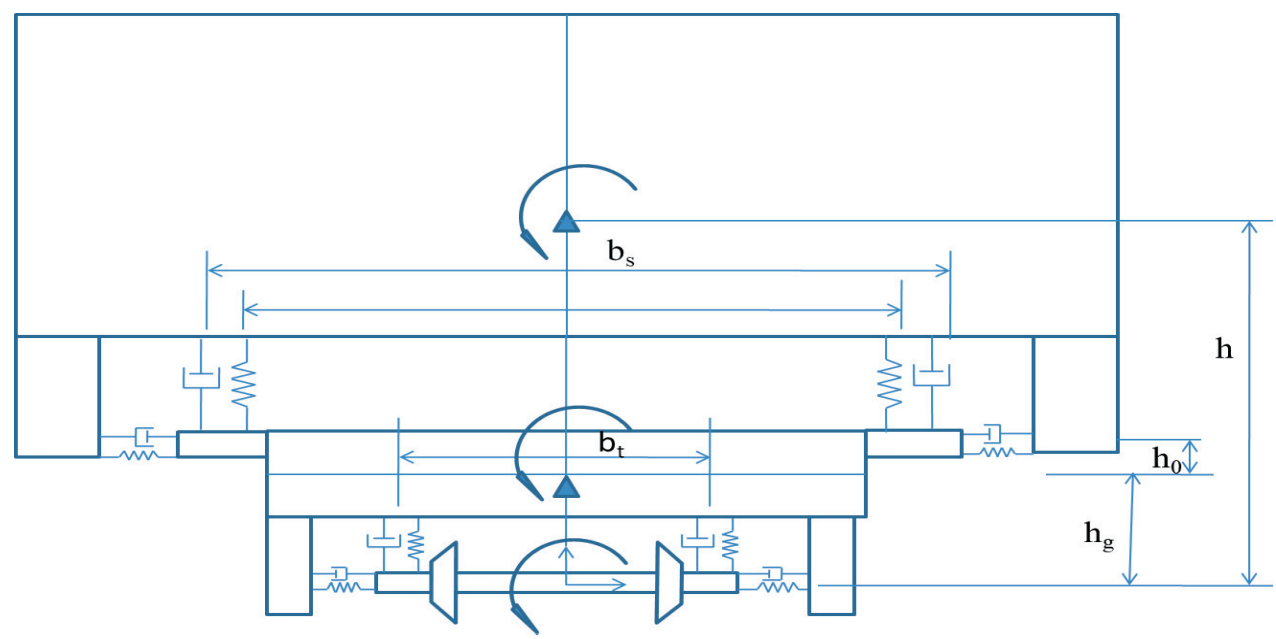

(b)

Figure 1. Schematic diagram of HSRV (a) top view (b) front view 


\section{Bondgraph Model of Carbody}

The bondgraph model exhibited in the Figure 2, is the sub-model of a vehicle car body of a railway vehicle system. A 0 -junction in the model is the joint between the parts connected to each other and represents force shared between them. A 1-junction in the model represents the velocity shared between all the connected parts at the 1-junction. The TF symbol represents the Transformer module. It represents the relation between flow in-flow out and force in-force out. The symbol $\mathrm{C}$ with complete arrow represents the signal bond. The function of the $\mathrm{C}$ symbol is to store the potential energy generated by the respective displacements. The symbol SE in the bondgraph represents the source of effort. It is included at the 1-junction of the bondgraph to integrate the force of gravity and centrifugal force of carbody's centre of gravity in vertical (z) and lateral (y) directions. In Bondgraph modelling technique, the force of suspension system is the interior part of the model and hence there is no need of representing suspension forces separately in a bondgraph model.

$$
\begin{aligned}
& \dot{Z}_{c f l}=\dot{Z}_{c}+l_{c} \dot{\phi}_{c}+l_{s} \dot{\theta}_{c} \\
& \dot{Y}_{c f l}=\dot{Y}_{c}+H_{1} \dot{\phi}_{c}+l_{s} \dot{\theta}_{c} \\
& \dot{Z}_{c f r}=\dot{Z}_{c}+l_{c} \dot{\phi}_{c}-l_{s} \dot{\theta}_{c} \\
& \dot{Y}_{c f r}=\dot{Y}_{c}+H_{1} \dot{\phi}_{c}+l_{s} \dot{\theta}_{c} \\
& \dot{Z}_{c r l}=\dot{Z}_{c}-l_{c} \dot{\phi}_{c}-l_{s} \dot{\theta}_{c} \\
& \dot{Y}_{c r l}=\dot{Y}_{c}+H_{1} \dot{\phi}_{c}-l_{s} \dot{\theta}_{c} \\
& \dot{Z}_{c r r}=\dot{Z}_{c}+l_{c} \dot{\phi}_{c}-l_{s} \dot{\theta}_{c} \\
& \dot{Y}_{c r r}=\dot{Y}_{c}+H_{1} \dot{\phi}_{c}-l_{s} \dot{\theta}_{c}
\end{aligned}
$$

The vehicle car body is considered as a rigid element. It is linked with the bogie via secondary suspension system at different locations. A kinematic relationship is formed between the mass centre and component of rotational velocity to express the velocities at different locations on a vehicle car body. These kinematic relations are expressed in equations 1 to 8 .

$$
\begin{gathered}
m_{c} \ddot{z}_{c}=F_{s f c z}-m_{c} g-\frac{m_{c} V^{2} \phi_{s e}}{R_{r}} \\
m_{c} \ddot{y}_{c}=F_{s f c y}+\frac{m_{c} V^{2}}{R_{r}}-m_{c} g \phi_{s e} \\
I_{c z} \ddot{\Psi}_{c}=M_{s c z} \\
I_{c y} \ddot{\theta}_{c}=M_{s c y} \\
I_{c x} \ddot{\phi}_{c}=M_{s c x}
\end{gathered}
$$




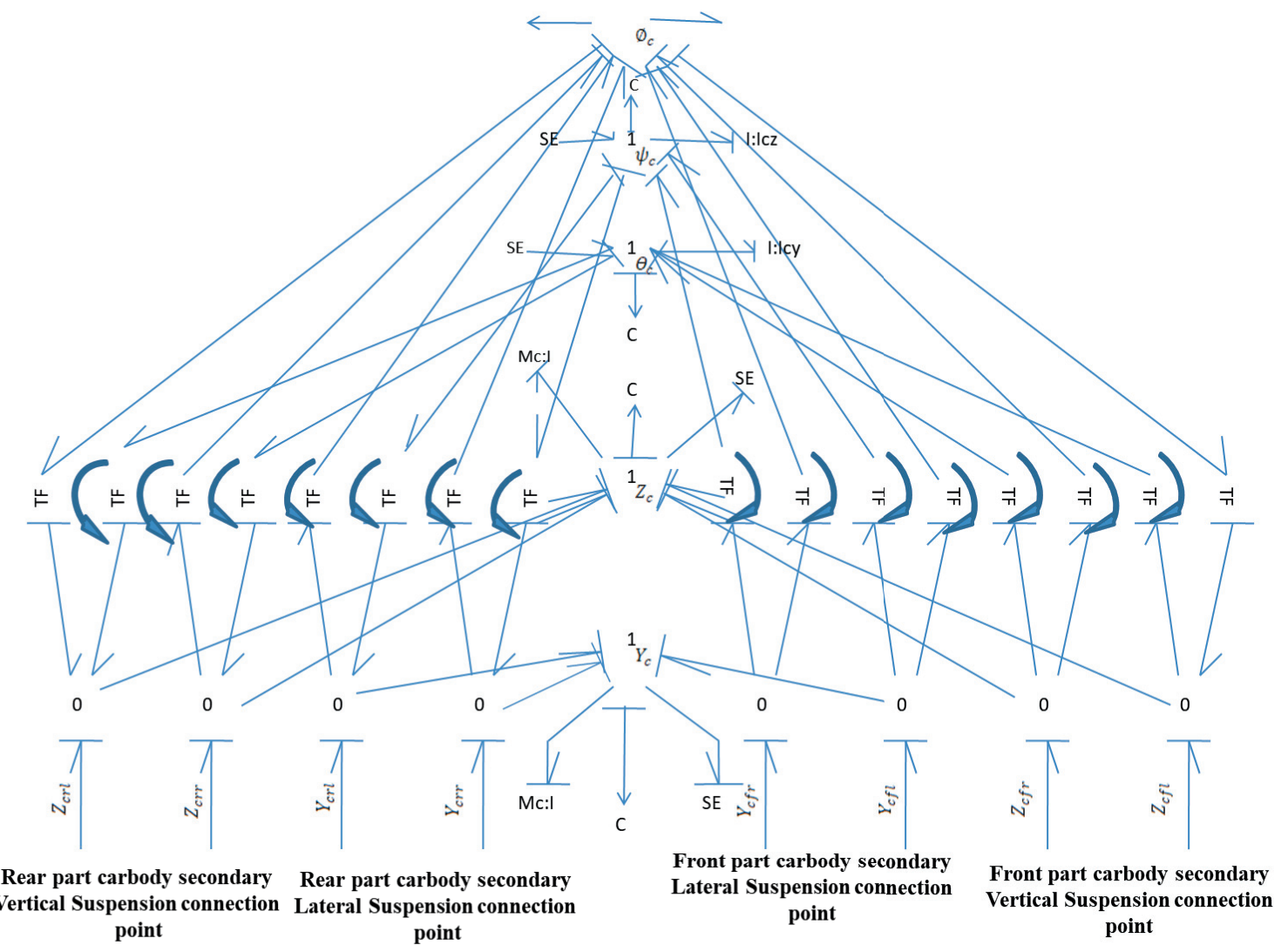

Figure 2. Bondgraph model of Carbody

Where $\varphi_{\text {se }}$ is the angle of super elevation, $\mathrm{R}_{\mathrm{r}}$ is curved radius. $\mathrm{F}_{\text {sfcz }}, \mathrm{F}_{\text {sfcy }}, \mathrm{M}_{\text {scz }}, \mathrm{M}_{\text {scy }}$, and $\mathrm{M}_{\text {scx }}$ are the suspension force and moment in the respective direction. Mathematical equation terms mentioned from 9 to 13 are the governing equations of a car body in a differential form. These equations are determined in vertical direction and lateral direction for roll, yaw and pitch motions.

\section{Bondgraph Model of Truck Frame}

The truck frame is considered as a rigid element of the railway vehicle system. Truck frame is coupled with the car body via secondary suspension system at different locations and also connected to selfsame wheelsets via primary suspension system as exhibited in figure 1. The kinematic relations shown in Equations 14 to 25 are employed to derive the velocity components in the corresponding directions at different connected locations. The bondgraph model of truck frame is shown in Figure 3.

$$
\begin{gathered}
\dot{Z}_{b l}=\dot{Z}_{b}-l_{p} \dot{\phi}_{b} \\
\dot{Y}_{b l}=\dot{Y}_{b}-h_{0} \dot{\phi}_{b} \\
\dot{Z}_{b r}=\dot{Z}_{b}+l_{p} \dot{\phi}_{b}
\end{gathered}
$$




$$
\begin{gathered}
\dot{Y}_{b r}=\dot{Y}_{b}-h_{0} \dot{\phi}_{b} \\
\dot{Z}_{b f l}=\dot{Z}_{b}-l_{p} \dot{\phi}_{b}+l_{t} \dot{\theta}_{b} \\
\dot{Y}_{b f l}=\dot{Y}_{b}-h_{0} \dot{\phi}_{b}+l_{t} \dot{\psi}_{b} \\
\dot{Z}_{b f r}=\dot{Z}_{b}+l_{p} \dot{\phi}_{b}+l_{t} \dot{\theta}_{b} \\
\dot{Y}_{b f r}=\dot{Y}_{b}+h_{g} \dot{\phi}_{b}+l_{t} \dot{\psi}_{b} \\
\dot{Z}_{b r l}=\dot{Z}_{b}-l_{p} \dot{\phi}_{b}-l_{t} \dot{\theta}_{b} \\
\dot{Y}_{b r l}=\dot{Y}_{b}+h_{g} \dot{\phi}_{b}-l_{t} \dot{\psi}_{b} \\
\dot{Z}_{b r r}=\dot{Z}_{b}-l_{p} \dot{\phi}_{b}+l_{t} \dot{\theta}_{b} \\
\dot{Y}_{b r r}=\dot{Y}_{b}+h_{g} \dot{\phi}_{b}-l_{t} \dot{\psi}_{b}
\end{gathered}
$$

Mathematical equation terms mentioned from 26 to 30 are the governing equations of a truck frame in a differential form.

$$
\begin{gathered}
m_{t} \ddot{y}_{t}=F_{s f t y}+\frac{m_{t} V^{2}}{R_{r}}-m_{t} g \Phi_{s e} \\
m_{t} \ddot{z}_{t}=F_{s f t z}-m_{t} g-\frac{m_{t} V^{2} \Phi_{s e}}{R_{r}} \\
I_{t z} \ddot{\Psi}_{t}=M_{s t z} \\
I_{t y} \ddot{\theta}_{t}=M_{s t y} \\
I_{t x} \ddot{\phi}_{t}=M_{s t x}
\end{gathered}
$$

\section{Bondgraph Model of Wheelsets}

The wheel set is a component of railway vehicle system which provides support to complete vehicle. A rigid wheelset in contact with the flexible Knife edged rail is considered for the present research work. The selfsame wheelsets were interconnected to the bogey through a primary suspension system. The bondgraph model of the selfsame wheelset is exhibited in Figure 4. Mathematical equation terms mentioned from 31 to 34 are the governing equations of a wheelset in a differential form. 


$$
\begin{gathered}
m_{w} \ddot{y}_{w}=\frac{m_{w} V^{2}}{R_{r}}-m_{w} g \phi_{s e}+F_{w L y}^{n}+F_{w R y}^{n}+N_{w y}^{L}+N_{w y}^{R}+F_{s f w y}-F_{w f c} \\
I_{w z} \ddot{\Psi}_{w}=-I_{w y} \frac{V}{R_{0}} \dot{\phi}_{w}+R_{x}^{R} F_{w R y}^{n}-R_{y}^{R} F_{w R x}^{n}+R_{x}^{L} F_{w L y}^{n}-R_{y}^{L} F_{w L x}^{n}+R_{x}^{R} N_{w y}^{R}+R_{x}^{L} N_{w y}^{L}+M_{w L z}^{n}+M_{s m w z} \\
+M_{w R z}^{n} \\
m_{w} \ddot{z}_{w}=-\frac{m_{w} V^{2} \phi_{s e}}{R_{r}}-m_{w} g+F_{w L z}^{n}+F_{w L z}^{n}+N_{w z}^{L}+N_{w z}^{R}+F_{s f w z} \\
I_{w x} \ddot{\phi}_{w}=-\frac{I_{w y} V\left(\frac{V}{R_{r}}-\dot{\Psi}_{w}\right)}{R_{0}}+R_{y}^{R} F_{w R z}^{n}-R_{z}^{R} F_{w R y}^{n}+R_{y}^{L} F_{w L z}^{n}-R_{z}^{L} F_{w L y}^{n}+R_{y}^{L} N_{w z}^{L}+R_{y}^{R} N_{w z}^{R} \\
-R_{z}^{L} N_{w y}^{L}-\quad R_{w y}^{R}+M_{w x}^{L}+M_{w x}^{R}+M_{s m w x}
\end{gathered}
$$

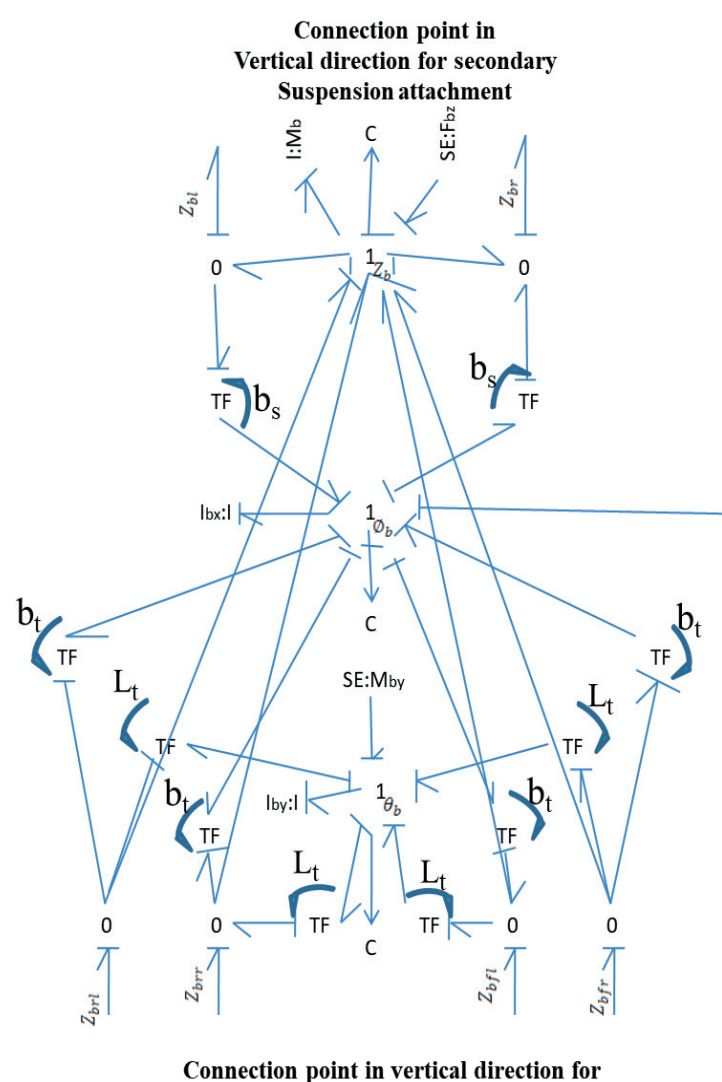

Primary suspension attachment

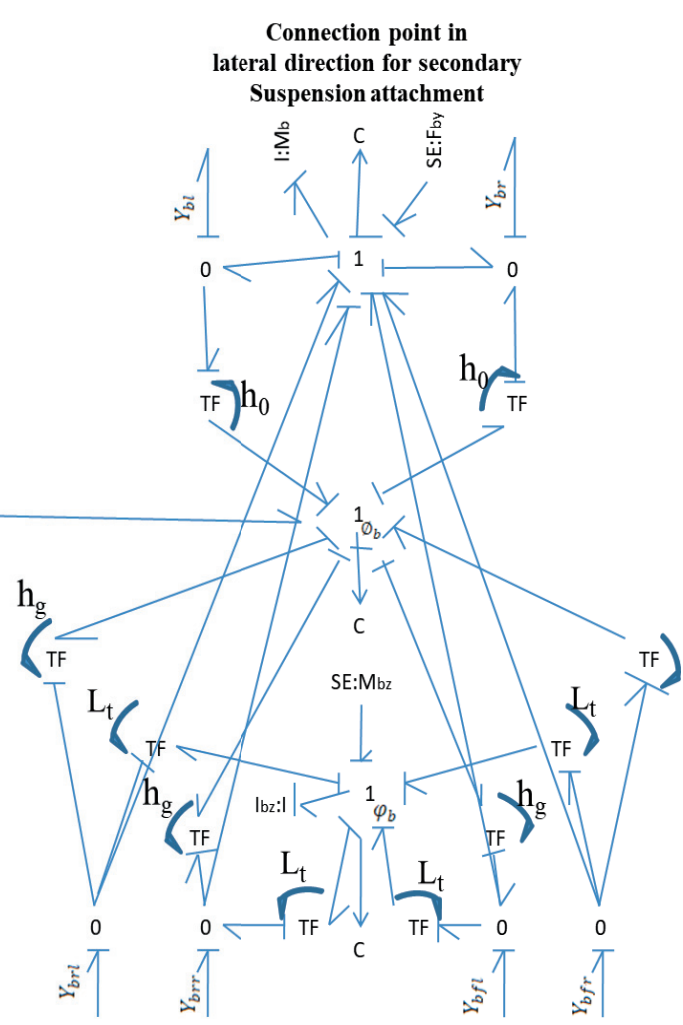

Connection point in lateral direction for Primary suspension attachment

Figure 3. bodgraph model of truckframe 
$F_{S f w z}, F_{s f w y}, M_{s m w z}$ and $M_{s m w y}$ are suspension forces and suspension moment of wheelset in corresponding direction. $F_{w L z}^{n}, F_{w L y}^{n}, F_{w L x}^{n}, M_{w x}^{L}, M_{w z}^{L}$ are the nonlinear creep forces and moment at the contact point on the left sided wheel-rail in the corresponding direction. $F_{w R z}^{n}, F_{w R y}^{n}, F_{w R x}^{n}, M_{w x}^{R}, M_{w z}^{R}$ are the nonlinear creep forces and moment at the contact point on the right sided wheel-rail in the corresponding direction. $N_{w y}^{L}, N_{w z}^{L}, N_{w y}^{R}, N_{w z}^{R}$ are the normal force component on left and right wheel-rail contact point. $R_{z}^{L}, R_{y}^{L}, R_{x}^{L} R_{z}^{R}, R_{y}^{R}, R_{x}^{R}$ are the position vector on the left and right contact point in respective direction. is the $F_{w f C}$ flange contact force, $x$ indicates longitudinal direction, $y$ indicates lateral direction and $z$ indicates vertical direction.

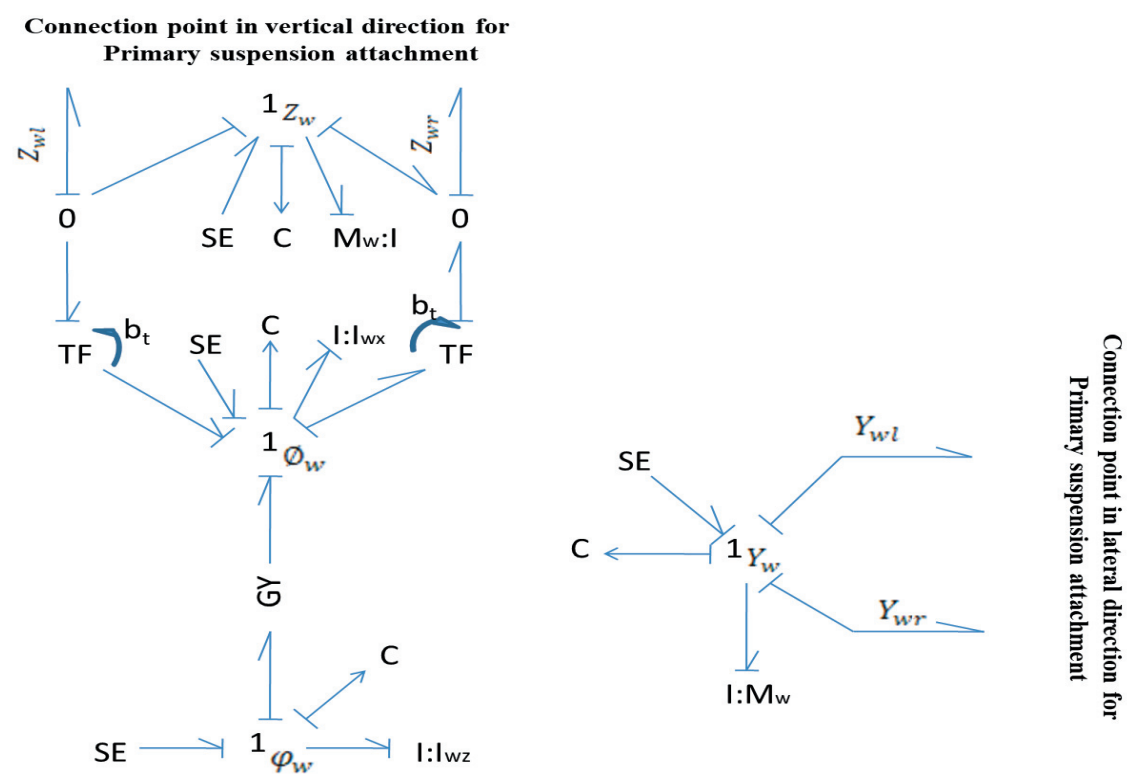

Figure 4: Complete bondgraph model of wheelset

\section{Heusristic Nonlinear Creep Model}

In the present work, Heuristic nonlinear creep model is utilised to form the contact forces between rails and wheels. Heuristic non-linear creep model is a combination of Kalker's linear creep theory and creep force saturation. The nonlinear creep forces and nonlinear creep moments are resulted by multiplying saturation factor to the corresponding linear creep force and linear creep moment as shown in the Equations 35 to 40.

$$
\begin{array}{ll}
F_{w L x}^{n}=\alpha_{i j} F_{w l x}^{L} & 35 \\
F_{w R x}^{n}=\alpha_{i j} F_{w r x}^{R} & 36 \\
F_{w L y}^{n}=\alpha_{i j} F_{w l y}^{L} & 37 \\
F_{w R y}^{n}=\alpha_{i j} F_{w r y}^{R} & 38
\end{array}
$$




$$
\begin{aligned}
& M_{w L z}^{n}=\alpha_{i j} M_{w l z}^{L} \\
& M_{w R z}^{n}=\alpha_{i j} M_{w r z}^{R}
\end{aligned}
$$

Where $F_{w l x}^{L}, F_{w l y}^{L}, M_{w l z}^{L}, F_{w r x}^{R}, F_{w r y}^{R}, M_{w r z}^{R}$ are linear creep forces and moment on left and right wheel in respective direction. Equations of linear creep forces and moments are taken from Kim et.al (2011). $\alpha_{i j}$ represent the saturation constant in the heuristic creep model obtained from Johnson's approach (Dukkipati, 2000) as given in equation 41.

$$
\alpha_{i j}=\left\{\begin{array}{c}
\frac{1}{\beta_{i j}}\left(\beta_{i j}-\frac{1}{3} \beta_{i j}^{2}+\frac{1}{27} \beta_{i j}^{3}\right)=>\beta_{i j} \leq 3 \\
\frac{1}{\beta_{i j}}=>\beta_{i j} \geq 3
\end{array}\right.
$$

Where $\beta_{i j}$ is the non linearity factor in heuristic creep model and it is calculated for vehicle under quasi-static motion over the curved track (Kim et.al).

\section{SIMULATION RESULTS}

\section{Derailment Analysis}

For many researchers, the derailment of railway vehicle is a cause of concern. The dynamic behaviour of a railway vehicle is impacted by its safety during the running operations. In order to investigate the operational safety of a running railway vehicle, a quotient is calculated and also observed. This quotient is known as Derailment quotient (DQ). It is the ratio of lateral and vertical forces generated in-between the wheels and rails. The limiting value of DQ is 0.8 when the wheel flange made contact with face of a rail (H Ishida et al., 1999). The maximum limit of DQ is 1.2 when the quasi-static testing is performed (SB Guarro., 1994).

The simulation has been performed using 'Symbol Shakti' to calculate the nonlinear creep forces in lateral and vertical direction. All the parameters of HSRV are taken from Kim et. Al (2011). While conducting the simulation, the impact of nonlinearities i.e. flanges contact and Heuristic nonlinear creeps were considered. The DQ can be comprehensively derived while taking the DOF of a wheelset model and nonlinear creep model into consideration. In the present study, all the criterions were fulfilled and the Derailment quotient is determined from the Equation 42 given below (H Ishida et al., 1999).

$$
\frac{Y_{L}}{Q_{L}}=\frac{F_{w L y, 11}^{n}+N_{w y, 11}^{L}}{F_{w z, 11}^{L}+N_{w z, 11}^{L}}
$$

Where $Y_{L}$ represents the lateral force acting on the left side wheel rail contact point, $Q_{L}$ is the vertical contact force working on the left wheel. A numerical technique called Runge-Kutta fourth order method has been used to evaluate the nonlinear contact forces in the time domain. This is done to investigate the time responses for the wheel set. For stability, the length of the time step is taken as $0.005 \mathrm{sec}$ in Runge-Ktutta fourth order method. The RMS values of 
derailment quotients have also been calculated. Figure 5 shows the comparison between the DQ evaluated from both linear and nonlinear creep model at $R_{r}=6000 \mathrm{~m}$.

In the present study, a perfect conical trade profiled wheel is employed for the calculation of derailment quotient. The conicity value of the wheel is considered to be constant and the value is mentioned in Table 1 of appendix 2 . The DQ is then calculated by using equation 42. Figure 6 to 9 shows the impact of vehicle speed on DQ with various suspension parameters of a 31 DoF railway model.

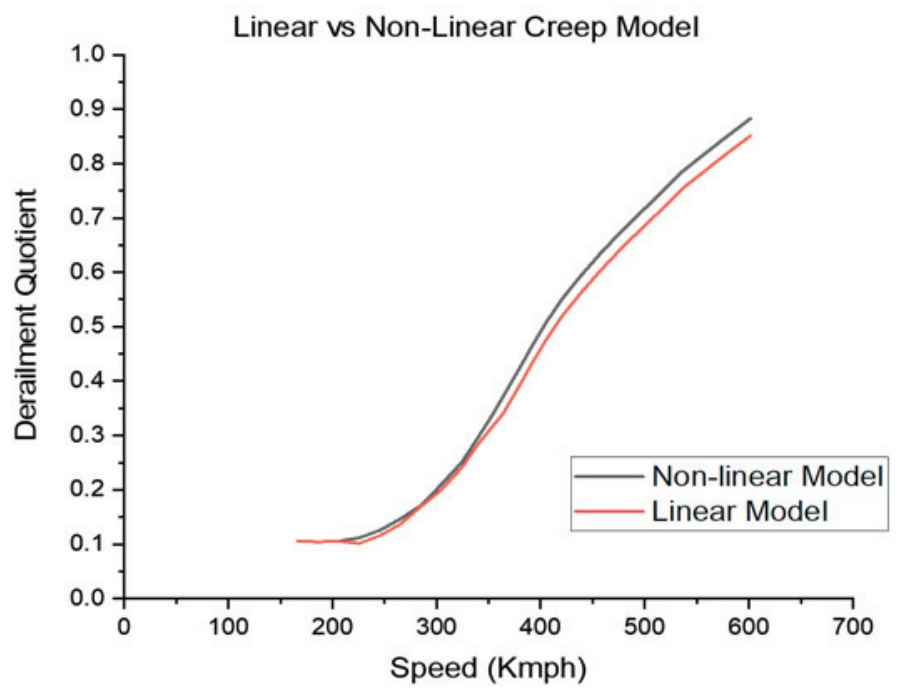

Figure 5. Comparison of linear and non linear model of 31 DOF high speed railway vehicle

Figure 6 represents the variation of DQ with respect to vehicle running speed of a 31 DOF model valuated by heuristic nonlinear creep model employed with soft and hard damping in lateral direction $\left(\mathrm{C}_{\mathrm{sy}}\right)$ of secondary suspension system. It is observed that the DQ have direct relation with vehicle running speed i.e. with increase of vehicle running speed, the DQ also increases. In a region where the running speed is smaller than $300 \mathrm{kmph}$, the gap between the evaluated DQ for hard damping and soft damping is small in the lateral direction. For vehicle speed more than 300 $\mathrm{kmph}$, the value of derailment quotient calculated using hard damper $C_{s y}$ is always more than that of calculated by using soft damper $C_{s y}$.

Figure 7 represents the variation of DQ with respect to vehicle running speed of a 31 DOF model valuated by heuristic nonlinear creep model employed with soft and hard damping in vertical direction $\left(\mathrm{C}_{\mathrm{sz}}\right)$ of secondary suspension system. It is observed that, for different values of $C_{s z}$, the DQ increases with increasing vehicle running speed. From Figure 7(a) and (b), it is observed that derailment quotients for hard vertical damper are greater than that of calculated for soft vertical damper when the vehicle running speed is more than $475 \mathrm{kmph}$. 


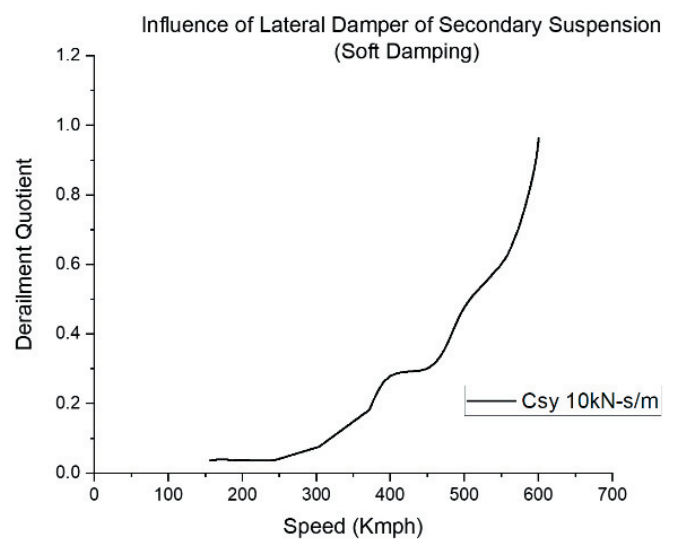

(a)

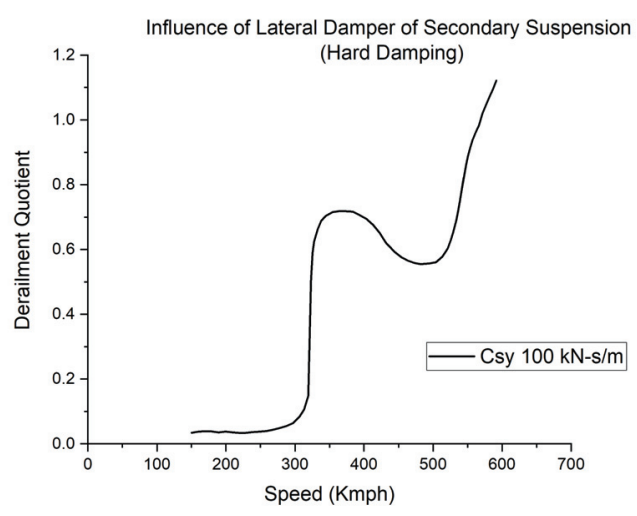

(b)

Figure 6. Variation of DQ with respect to vehicle running speed for secondary suspension system (a) for soft lateral damping (b) for hard lateral damping.

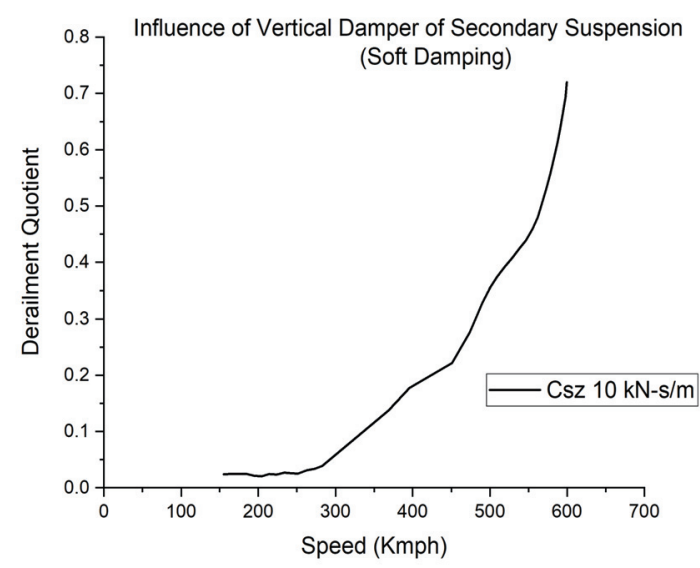

(a)

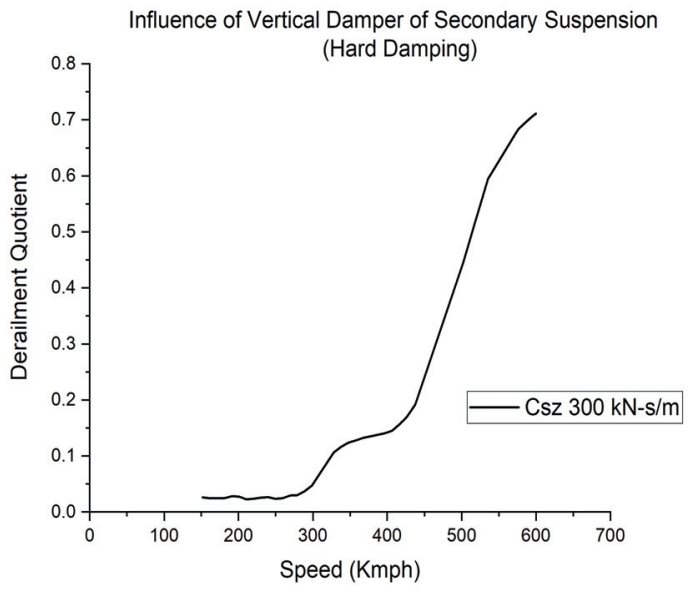

(b)

Figure 7. Variation of DQ with respect to vehicle running speed for secondary suspension system

(a) for soft vertical damping (b) for hard vertical damping.

Figure 8 represents the variation of DQ with respect to vehicle running speed of a 31 DOF model valuated by heuristic nonlinear creep model employed with soft and hard stiffness in lateral direction $\left(K_{p y}\right)$ of primary suspension system. It is observed that the DQ have direct relation with vehicle running speed i.e. with increase of vehicle running speed, the DQ also increases. For the nonlinear creep model, the DQ evaluated by using soft lateral stiffness $K_{p y}$ is always more than the value calculated using hard lateral stiffness $K_{p y}$. When vehicle running speed is more than $440 \mathrm{kmph}$, the value of derailment quotient calculated for hard stiffness $K_{p y}$ is always be more than the value of derailment quotient calculated for soft stiffness $K_{p y}$. Comparing results of Figure 9(a) and 9(b), it has been observed that derailment quotient of hard vertical primary suspension is greater compare to soft suspension when the vehicle running speed is more than $475 \mathrm{kmph}$. 


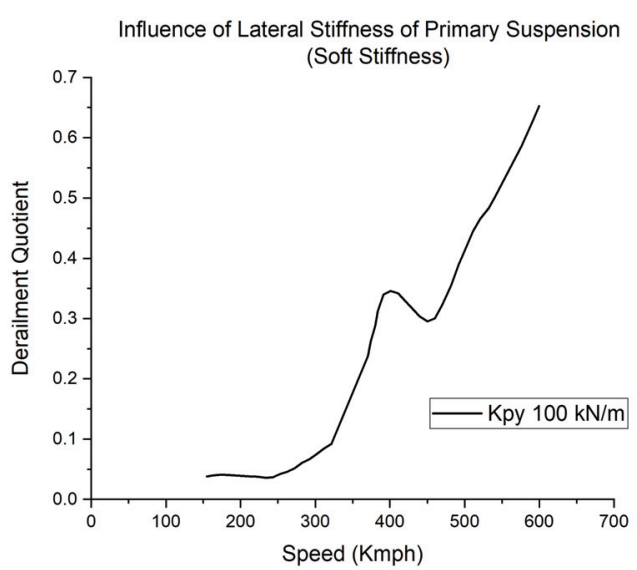

(a)

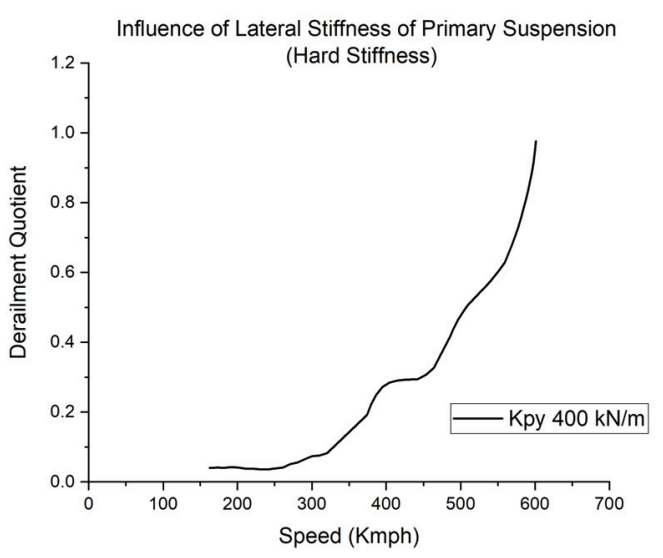

(b)

Figure 8. Variation of DQ with respect to vehicle running speed for primary suspension system (a) for soft lateral stiffness (b) for hard lateral stiffness.

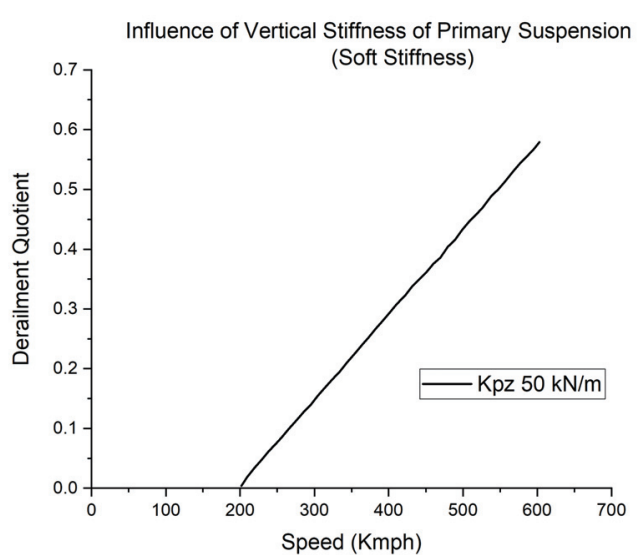

(a)

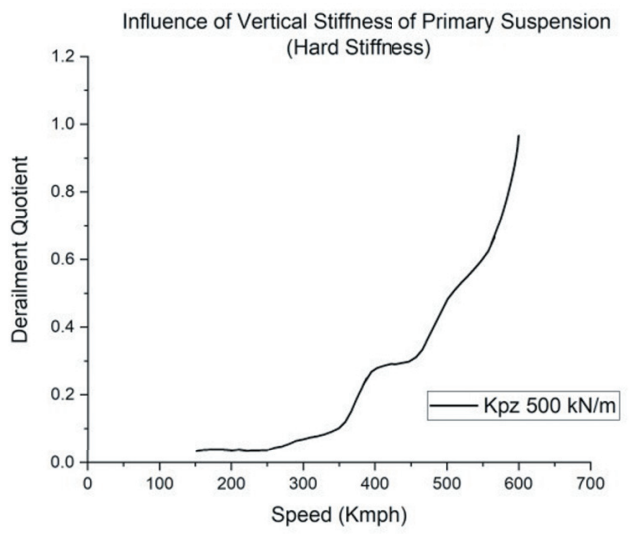

(b)

Figure 9. Variation of DQ with respect to vehicle running speed for primary suspension system (a) for soft vertical stiffness (b) for hard vertical stiffness.

\section{CONCLUSIONS}

In the presented work, the dynamic response of HSRV travelling on a curved track has been analysed by utilising Heuristic nonlinear creep model. A 31 DOF full car model was developed to completely describe the dynamics of a railway vehicle. A perfectly conical wheel profile has been used for determining of derailment quotient. The impact of various damping and stiffness parameters on derailment quotient has also been observed. 
The following points were concluded from the presented research work:

1. The derailment quotient (DQ) is having a direct relation with the vehicle running speed. As the running speed increases, the DQ also increases.

2. The derailment quotients valuated by incorporating nonlinear creep model are more than the derailment quotient valuated by linear creep model.

3. In the primary suspension system of a nonlinear creep model, a spring of soft stiffness $\left(\mathrm{K}_{\mathrm{pz}}=50 \mathrm{kN} / \mathrm{m}\right)$ produce lower derailment quotient in the vertical direction than a spring of Hard stiffness $\left(\mathrm{K}_{\mathrm{pz}}=500 \mathrm{kN} / \mathrm{m}\right)$. The similar relation has also been observed in the lateral direction stiffness $\left(\mathrm{K}_{\mathrm{py}}\right)$ of primary suspension system.

4. In secondary suspension system of nonlinear creep model, the damper of soft damping $\left(\mathrm{C}_{\mathrm{sz}}=10 \mathrm{kN}-\mathrm{s} / \mathrm{m}\right)$ produce lower derailment quotient in the vertical direction than a damper of hard damping $\left(\mathrm{C}_{\mathrm{sz}}=300 \mathrm{kN}-\mathrm{s} / \mathrm{m}\right)$. The similar relation has also been observed in lateral direction damping $\left(\mathrm{C}_{\mathrm{sy}}\right)$ of secondary suspension system.

5. The change in the vehicle running speed also has an impact on the derailment quotient while running on a sharp curved track. The vehicle running at a low speed generates greater derailment quotient valuated from nonlinear creep model than compared to the derailment quotient valuated from linear creep model.

\section{REFERENCES}

F.J Nadal, 1896. Theorie de la stabilite des locomotives. Vvec.dunod.

H. Weinstock, 1984. Wheel climb derailment criteria for evaluation of rail vehicle safety. ASME: 1 .

L.M. Sweet, A. Karmel \& P.K. Moy, 2018. Wheel climb Derailment Criteria Under Steady Rolling and Dynamic Loading Conditions. The Dynamics of Vehicles on roads and on tracks. Routledge: 496-510.

R.S. Barbosa, 2004. A 3D Contact Force Safety Criterion for Flange Climb Derailment of a Railway Wheel. Vehicle System Dynamics, 42(5):289-300.

C.E. Bell \& J.K. Hedrick, 1981. Forced Steering of Rail Vehicles: Stability and Curving Mechanics. Vehicle System Dynamics, 10(6):357-86.

Y.C Cheng \& C.T Hsu, 2011. Hunting stability and derailment analysis of a car model of a railway vehicle system. Proceedings of the Institution of Mechanical Engineers, Part F: Journal of Rail and Rapid Transit. SAGE Publications, 226(2):187-202.

R.V Dukkipati \& S.S Narayana, 2001. Lateral stability and steady state curving performance of unconventional rail trucks. Mechanism and Machine Theory, 36(5):577-87.

A.H Wickens, 1996. Railway Vehicles with Generic Bogies Capable of Perfect Steering. Vehicle System Dynamics, 25(6):389-412.

W. Wang \& G. Li, 2012. Development of high-speed railway vehicle derailment simulation - Part II: Exploring the derailment mechanism. Engineering Failure Analysis, 24:93-111.

K. Zboinski \& M. Dusza, 2006. Development of the method and analysis for non-linear lateral stability of railway vehicles in a curved track. Vehicle System Dynamics, 44(sup1):147-57.

J. Zeng \& P. Wu, 2004. Stability analysis of high speed railway vehicles. JSME int. j, 47(2):464-470.

O. Polach, 2006. On non-linear methods of bogie stability assessment using computer simulations. Proceedings of the Institution of Mechanical Engineers, Part F: Journal of Rail and Rapid Transit, 220(1):13-27. 
O. Polach, 1999. A Fast Wheel-Rail Forces Calculation Computer Code. Vehicle System Dynamics, 33(sup1):728-39.

Y.C Cheng, S.Y Lee \& H.H Chen, 2009. Modelling and nonlinear hunting stability analysis of high-speed railway vehicle moving on curved tracks. Journal of Sound and Vibration, 324(1-2):139-60.

W. Wang \& G.X Li, 2010. Development of a simulation model of a high-speed vehicle for a derailment mechanism. Proceedings of the Institution of Mechanical Engineers, Part F: Journal of Rail and Rapid Transit, 224(2):10313.

M. Durali \& M.M Jalili, 2009. A new criterion for assessment of train derailment risk. Proceedings of the Institution of Mechanical Engineers, Part K: Journal of Multi-body Dynamics, 224(1):83-101.

H. Ishida \& M. Matsuo, 1999. Safety Criteria for Evaluation of Railway Vehicle Derailment. Quarterly Report of Railway Technical Research Institute, 40(1):18-25.

S.B Guarro, 1994. Reliability, availability, maintainability and safety assessment. Reliability Engineering \& System Safety, 43(3):331-2.

R.V.Dukkipati, 2000. “vehicle dynamics," crc press.

Kim P, Jung J and Seok J. 2011. A parametric dynamic study on hunting stability of full dual-bogie railway vehicle. International Journal of Precision Engineering and Manufacturing; 12: 505-519. 